\title{
Investigation of natural and artificial stimulation of the Ionospheric Alfvén Resonator at high latitude
}

\author{
T. K. Yeoman ${ }^{\mathrm{a}}$, H. C. Scoffield ${ }^{\mathrm{a}}$, D. M. Wright ${ }^{\mathrm{a}}$, L. J. Baddeley ${ }^{\mathrm{a}}$, A. N. Vasilyev ${ }^{\mathrm{b}}$ and N. V. \\ Semenova ${ }^{\mathrm{b}}$ \\ ${ }^{a}$ Department of Physics and Astronomy, University of Leicester, LE1 7RH, UK. \\ ${ }^{b}$ Polar Geophysical Institute, Apatity, Murmansk region 184209, Russia
}

\begin{abstract}
A brief review is provided of recent progress in understanding the Ionospheric Alfvén Resonator (IAR) at high latitude. Firstly, naturally-occurring resonances of the IAR as detected by pulsation magnetometers in the auroral zone at Sodankylä and in the polar cap at Barentsburg are considered. The characteristics of the IAR in the two regions are broadly similar, although the effects of solar illumination are less clear at the higher latitudes. Secondly we review recent attempts to stimulate the IAR through high-power radio frequency experiments both in the auroral zone at Tromsø with the European Incoherent SCATter (EISCAT) heater, and within the polar cap at Longyearbyen with the Space Plasma Exploration by Active Radar (SPEAR) facility. In the auroral zone at, Tromsø the stimulated IAR has been observed by ground-based magnetometers, and through electron acceleration observed on the FAST spacecraft. At SPEAR in the polar cap, the stimulated IAR has been investigated, with ground magnetometers, with the first results indicative of a positive detection.
\end{abstract}

\section{Introduction}

The Ionospheric Alfvén Resonator (IAR) results from the vertical structure associated with the decay in plasma density going from the ionosphere to the magnetosphere, whose existence was first predicted by Polyakov, (1976). The lower boundary region occurs in the ionospheric F-layer. The upper boundary of the IAR corresponds to the region where Alfvén waves propagating along the geomagnetic field lines are partially reflected by large Alfvén velocity gradients, caused by the rapid decrease in plasma density above the F-layer density peak. At this upper boundary (defined by a sharp peak in the Alfvén velocity profile at an altitude of $\sim 0.5 R_{E}$ ) energisation of electrons occurs, which ultimately leads to the excitation of aurora. These energised particles can also be detected by orbiting spacecraft. As such the IAR holds great promise for revealing important physics in magnetosphere-ionosphere coupling and auroral particle acceleration. The characteristic signatures of the resonant IAR cavity were first observed by Belyaev et al. (1987) in the form of multiple horizontally banded spectral resonance structures (SRS) in dynamic spectra of mid-latitude magnetic background noise between $0.1 \mathrm{~Hz}$ and $10 \mathrm{~Hz}$ derived from pulsation magnetometer data. Such signatures are weak, and easily masked by other wave phenomena, but may be observed over several hours on geomagnetically quiet days. Strong diurnal control of the SRS signatures is usually observed, with the lowest frequencies observed when solar illumination and hence ionospheric density are the strongest. Here we review recent progress in understanding the IAR at high latitude. This review is divided into four sections; naturally occurring resonances in the auroral zone; naturally occurring resonances within the polar cap; artificial stimulation of the IAR in the auroral zone; and finally artificial stimulation of the IAR within the polar cap.

\section{Natural resonances of the IAR at high latitude}

\subsection{The auroral zone}


IAR resonance features at high latitudes were first observed as SRS signatures between 0.2 and 5 $\mathrm{Hz}$ by Belyaev et al., (1999). In order to understand the controlling factors in the observed SRS frequencies, Hebden et al. (2005) investigated the evolution of the resonant frequencies with local time for days when they were clearly visible. Spectra from a whole month of pulsation magnetometer data from Sodankylä, Finland were considered, during which ten clear intervals of SRS were identified. Dynamic spectra of the magnetometer data were produced by applying a Fast Fourier Transform (FFT) to a sliding window of $100 \mathrm{~s}$ of data, with a slip of $100 \mathrm{~s}$, producing a frequency resolution of $0.01 \mathrm{~Hz}$ and a time resolution of $100 \mathrm{~s}$. Figure 1 presents an average of 24 such spectra, in order to highlight persistent SRS features over more ephemeral noise signals, reducing the time resolution to $40 \mathrm{~min}$.

When the diurnal variation of the frequencies of the SRS signatures such as those illustrated in Figure 1 were examined, a strong linear relationship was found between harmonic frequency and harmonic number, consistent with the simple 1-D vertical model of Trakhtengerts et al. (2000), in which the IAR consists of 3 layers of constant density. The middle layer represents the standing wave cavity which is bounded below by a conduction layer and above by a low density layer leading to the wave trapping and resonance features of the IAR. This model was thus used for interpretation of the signatures. For resonances characterised by wave speed $v_{a}$, and cavity scale size $L$, the frequency $f$ of the harmonic signature number $N$ is given by

$$
f=\frac{(N+\Phi) v_{a}}{2 L}
$$

where $\Phi$ is the wave "phase factor" and $N=0,1,2 \ldots$ (based on Polyakov and Rapoport, 1981, but adopting the terminology used by Hebden et al., 2005). The value of $\Phi$ is controlled by the reflection coefficient at the lower IAR bounbdary, which is given by

$$
R=\frac{\Sigma_{w} / \Sigma_{P}-1}{\Sigma_{w} / \Sigma_{P}+1}
$$

where $\Sigma_{P}$ is the height integrated Pedersen conductivity and $\Sigma_{w}$ is the wave impedence (Lysak, 1991),

$$
\Sigma_{w}=\frac{1}{\mu_{o} v_{\mathrm{IAR}}} .
$$

For a wave node at the lower reflecting boundary, as expected for high $\Sigma_{P}$, then $R=-1$ and $\Phi$ will be half integer. Conversely, for a wave antinode at the lower reflecting boundary, as expected for low $\Sigma_{P}$, then $R=1$ and $\Phi$ will be integer (Lysak, 1991). The upper boundary is always an antinode. This arrangement is illustrated in Figure 2. Hebden et al. (2005) performed an analysis comparing model and data for 10 SRS intervals, and concluded that the average value of $\Phi$ was 0.61 , close to the value expected for the high Pedersen conductivity limit. An average value of the scale length of the cavity, $L$ was also calculated at $L=530 \mathrm{~km}, 2-3$ times the typical plasma scale height in the ionosphere. They concluded that changes in the observed SRS frequencies were dominated by changes in the ionospheric plasma density, and hence the Alfvén velocity, rather than changes in the cavity size or lower reflection boundary condition, which were considerably more stable with time of day.

\subsection{The polar cap (Barentsburg)}

Whilst the characteristics of the IAR at auroral latitudes have come under detailed scrutiny over recent years, the polar cap region is much less studied. Recently natural SRS have been observed in pulsation magnetometer data from Barentsburg, Svalbard at $L$-shells of 15 (Semenova et al., 2005). It was found that the characteristics of SRS within the polar cap were similar to those observed in the auroral zone and that they could be explained by similar IAR theory. An example of polar cap IAR signatures is presented in Figure 3. Observations from the D component on 15 August 2005 
are presented from the pulsation magnetometer of the Barentsburg Observatory of the Polar Geophysical Institute. Here a sampling rate of $40 \mathrm{~Hz}$ and a 1024 point FFT analysis provides a spectrum every $25.6 \mathrm{~s}$, with a frequency resolution of $0.04 \mathrm{~Hz}$. The top panel of Figure 3 presents averages of 20 such spectra, resulting in an 8.5 min FFT window, which is slipped by $25.6 \mathrm{~s}$ in the dynamic spectrum, and normalised to the peak power at $3 \mathrm{~Hz}$. Clear signatures of SRS are seen, but with less of a diurnal variation of the SRS frequencies than is observed in the auroral zone, as a result of the very high latitude and permanent solar illumination during August. As discussed in Section 2.1, the harmonic spacing of the SRS is vital when comparing observations with model results. The lower panel presents an analysis of the SRS signatures to determine the harmonic separation of the SRS in order to facilitiate such a comparison with IAR models. Here the averaged spectra in the upper panels are bandpass-filtered between $0.2-1 \mathrm{~Hz}$ in the frequency domain, and then subjected to an autocorrelation analysis. This clearly illustrates the frequency separation of the SRS harmonics as a function of time. Analysis of such polar cap data is ongoing, both in terms of natural resonances of the IAR, and artificial stimulation experiments, as reported in section 3 below.

\section{Artificial stimulation of the IAR}

\subsection{The auroral zone}

Figure 4 presents a summary of the first 10 years experiments aimed at the generation of artificial magnetic disturbances with the EISCAT heater. The average amplitude of EISCAT heater-induced artificial magnetic disturbances as detected on ground magnetometers in the vicinity of the heater is plotted as a function of the modulation frequency of the RF power. The induced signals are clearly strongest at the lower end of the frequency range, but significant signals are also indicated in the frequency range $1-10 \mathrm{~Hz}$, which covers the expected frequencies of the first few harmonics of the IAR.

Bösinger et al., (2000) reported on a series of experiments, using the high power RF facility at Tromsø, designed to produce artificial magnetic pulsations in the frequency range of the lower harmonics of the IAR. The experiments involved a stepwise sweep of the modulation frequency from approximately 0.1 to $3 \mathrm{~Hz}$. The results of one of these experiments are presented in Figure 5, where the artificial signal is clearly discernable above the background noise at frequencies of 1.5 $2.5 \mathrm{~Hz}$, from 12:40 - 12:55 UT. Although the sequence of experiments were of variable success, on one occasion a clear enhancement of power was observed in a narrow region of the modulation frequency sweep, centred at $1.9 \mathrm{mHz}$. This occurred at a time of day when IAR effects are generally clear, and was taken as evidence that the modulation frequency at $1.9 \mathrm{mHz}$ was close to a natural resonant frequency of the IAR, thus the response to the modulation was strongest at this frequency, and the IAR was driven to resonance by the high power ionospheric modification experiment.

Whilst Bösinger et al. (2000) investigated the ground magnetic signature of the IAR during artificial stimulation experiments, Cash et al. (2002) presented a quantitative analysis of the electric field and particle flux data from the Fast Auroral SnapshoT (FAST, Carlson et al., 1998) spacecraft in order to investigate the in situ particles and fields in the IAR. The spacecraft data were examined during a transit of the spacecraft footprint over the ionosphere overlying the Tromsø heater during a modulated X-mode heating experiment at $4.04 \mathrm{MHz}$, as described by Robinson et al., (2000). The Tromsø heater was transmitting at high power with a $3 \mathrm{~Hz}$ modulation, and this resulted in the launch of Alfvén waves from the lower ionosphere into the magnetosphere, allowing the first observations of electron acceleration within the IAR. Cash et al., (2002) established the timeevolution of the $3 \mathrm{~Hz}$ signals in the electric field, and the downgoing electron flux at 32.3, 64.7, and $129.4 \mathrm{eV}$. A clear dispersion was observed in the times of detection of the energetic electron signals, which was demonstrated to be consistent with an electron acceleration region between 3250 
and $3550 \mathrm{~km}$ altitude. A simple model of this acceleration region was developed in order to investigate the electron measurements on more detail. The altitude variation of the accelerating potential above the FAST altitude $\left(z_{1}\right)$ was assumed to be

$$
\varphi(z, t)=\varphi\left(z_{1}\right) \exp \left(\frac{z-z_{1}}{H}\right) \cos \omega t,
$$

where $H$ is the characteristic height of the plasma density inhomogeneity and $\omega$ the angular frequency of the $3 \mathrm{~Hz}$ wave. As the time taken for the electrons to traverse this region is short compared to the wave period, this potential was taken to be quasi-static, and would then shift the electron energy distribution according to

$$
f(E) \rightarrow f(E+\Delta E),
$$

where $f(E)$ is the phase space density and $\Delta E$ is the energy gained across the potential drop. For a particle distribution which follows a power law as

$$
\frac{f(E)}{f\left(E_{0}\right)}=\left(\frac{E}{E_{0}}\right)^{n},
$$

where $E_{0}$ is a constant, then the observed fluxes should be obtained by shifting the power law particle distribution by $\Delta E$. This process is detailed in Figure 6 for the three downgoing electron energy channels measured by FAST, and for three observed peaks in the oscillating electron fluxes observed during the experiment. The values of $\Delta E$ for each peak vary across the flux tube traversed by FAST in a fashion consistent with a potential with a horizontal Gaussian cross section of width $\sim 10 \mathrm{~km}$, implying parallel electric field strengths of $\sim 10^{-2} \mathrm{mVm}^{-1}$ (Cash et al., 2002).

\subsection{The polar cap}

Whilst well-established high power ionospheric modification facilities such as the EISCAT heater at Tromsø have demonstrated the ability to perform controlled active experiments on the IAR at auroral latitudes, as detailed in section 3.1, until recently it was not possible to do such experiments at higher latitudes on the polar cap. SPEAR (Space Plasma Exploration by Active Radar) is a new high-power radar system located at $78.15^{\circ} \mathrm{N}$ latitude, $16.05^{\circ} \mathrm{E}$ longitude, located adjacent to the EISCAT Svalbard Radar (ESR) in the vicinity of Longyearbyen (Spitzbergen) and is designed to carry out a range of space plasma investigations of the polar ionosphere and magnetosphere, including experiments to stimulate the IAR. The SPEAR antenna system comprises a $6 \times 4$ array of full-wave, crossed-dipoles, $16 \mathrm{~m}$ above the ground, with an antenna spacing of $48.4 \mathrm{~m}$, allowing the transmission of both linear and circularly polarised signals. The individual dipoles are rhombically broadened to allow operation between 4 and $6 \mathrm{MHz}$. The resulting beam has a quasi-elliptical crosssection, with an average half-power width of $21^{\circ}$ along its major axis and $14^{\circ}$ along its minor axis. This results in an overall antenna gain of $21 \mathrm{~dB}$. During the operations reported here the complete $6 \times 4$ SPEAR array was available (48 transmitters), operating at $4.45 \mathrm{MHz}$. The transmitters were operated at $2 \mathrm{~kW}$, resulting in an ERP for SPEAR of $\sim 15 \mathrm{MW}$. On 2 December 2005, as recently reported by Scoffield et al. (2006), a modulated X-mode ionospheric modification experiment was carried out at SPEAR, with the intention of artificially stimulating the Ionospheric Alfvén Resonator. A modulation frequency of $3 \mathrm{~Hz}$ was superimposed on a 20 minute on/off cycle. The magnetic field changes due to this experiment were recorded on the nearby Barentsburg (BAR) station of the University of Oulu pulsation magnetometer chain located at the Barentsburg Observatory of the Polar Geophysical Institute

Data from the BAR magnetometer are presented in dynamic spectral form in for the $\mathrm{H}$ and D component respectively in Figure $7 \mathrm{a}$ and $\mathrm{b}$. Each data point plotted represents the mean of 20 Fourier spectra, created from consecutive, independent 25.6 second (1024 point) spectral windows centred at that time. The slip distance between data points is 6.4 seconds (256 points). The 
frequency resolution of the dynamic spectra is $0.04 \mathrm{~Hz}$. In order to draw out SRS features each spectrum was filtered in the frequency domain. The mean power of each spectrum was removed and a Lanczos squared filter, with a high frequency cutoff of $1.0 \mathrm{~Hz}$ and a low frequency cutoff of 0.2 $\mathrm{Hz}$ was used. The colour scale shows the Fourier power as a fraction of the maximum power observed at $3 \mathrm{~Hz}$ during the interval. The bar at the top of Figure 7 and the vertical dashed lines indicate the times when SPEAR was turned on. The red shading of the bar indicates "on" and the blue shading indicates "off", with the intervals labelled 1 to 7 . The horizontal white lines mark the frequencies $2.9 \mathrm{~Hz}$ and $3.1 \mathrm{~Hz}$ where, if the ionospheric conditions are favourable, one might expect to see an enhancement in power at $3 \mathrm{~Hz}$ during the times when SPEAR is turned on (intervals 2, 4, and 6). Indeed there is an enhancement of the D component during interval 2 between 1800-1820 UT and also during interval 6 between 1920-1940 UT. During interval 4 (1840-1900 UT) the conditions become quite disturbed. No D component enhancement at $3 \mathrm{~Hz}$ is observed during this interval, but such an enhancement is seen in the $\mathrm{H}$ component. These enhancements may indicate artificial stimulation of the ionospheric Alfvén resonator at or near one of the harmonics of its resonant frequency. During interval 4 the IMAGE magnetometer data show a disturbance in the geomagnetic field at all stations, indicative of substorm activity. It is possible that the polarization of the oscillation is related to the background ionospheric electric fields, which have been altered by the disturbed conditions during intervals $3-5$. The magnetic field changes measured by IMAGE indicate a transient rotation of the ionospheric electric field during intervals $3-5$, as a westward electrojet is established.

\section{Summary}

Although first proposed some 30 years ago, by Polyakov (1976), and confirmed observationally a decade later by Belyaev et al. (1987), the existence of the ionospheric Alfvén resonator at high latitudes was only confirmed by Belyaev et al. (1999). The upper boundary of the IAR, where the Alfvén velocity profile reaches a maximum, is a region where electron energisation occurs, and thus holds important information on the physical processes involved in magnetosphere-ionosphere coupling and auroral particle acceleration. Since the first detection of the IAR considerable progress has been made in characterising and understanding the IAR, both in the auroral zone and the polar cap. A combination of pulsation magnetometer data and modelling have established the basic morphology of the IAR, and how it is controlled by the dimensions, boundary conditions and plasma density of the resonant cavity. Recent improvements in high latitude instrumentation will lead to further improvements in our understanding of this important magnetospheric region. Recent developments in active experiments designed to stimulate the IAR offer a new insight into the structure and physical processes of the IAR. Combined with spacecraft data such experiments offer the chance to explore the role of the IAR in magnetosphere-ionosphere coupling processes and auroral particle acceleration.

\section{Acknowledgments.}

SPEAR is supported by PPARC grant PP/E002544/1, HCS and LJB are supported by PPARC grant PPA/G/O/2003/00013. A.N.V. and N.V.S are supported by the RFBR grant 05-05-62414.

\section{References}

Belyaev, P. P., S. V. Polyakov, V. O. Rapoport, and V. Y. Trakhtengerts, Discovery of resonance structure in the spectrum of atmospheric electromagnetic background noise in the range of short-period geomagnetic pulsations, Dokl. Akad. Nauk SSSR, 297, 840- 846, 1987.

Belyaev, P. P., T. Bösinger, S. V. Isaev, and J. Kangas, First evidence at high latitudes for the ionospheric Alfvén resonator, J. Geophys. Res., 104, 4305-4317, 1999. 
Bösinger, T., T. Pashin, A. Kero, P. Pollari, P. Belyaev, M. Rietveld, T. Turunen, and J. Kangas, Generation of artificial magnetic pulsations in the Pc1 frequency range by periodic heating of the Earth's ionosphere: Indications of ionospheric Alfvén resonator effects, J. Atmos. Sol. Terr. Phys., 62, 277-297, 2000.

Carlson, C. W., Pfaff, R. F., and Watzin, J. G.: The Fast Auroral SnapshoT (FAST) mission, Geophys. Res. Lett., 25, 2013-2016, 1998.

Cash, S. R., J. A. Davies, E. Kolesnikova, T. R. Robinson, D. M. Wright, T. K. Yeoman, and R. J. Strangeway, Ann. Geophys., 20, 1499-1507, 2002.

Hebden, S. R., T. R. Robinson, D. M. Wright, T. K. Yeoman, T. Raita, and T. Bösinger, A quantitative analysis of the diurnal evolution of ionospheric Alfvén resonator magnetic resonance features and calculation of changing IAR parameters, Ann. Geophys., 23, 1711 1721, 2005.

Lysak, R. L., Feedback instability of the Ionospheric Resonant cavity, J. Geophys. Res., 96, 1553$1568,1991$.

Stubbe, P., Review of ionospheric modification experiments at Tromsø, J. Atmos. Terr. Phys., 58, 349-368, 1996.

Polyakov, S. V., On properties of an ionospheric Alfvén resonator, in Symposium KAPG on SolarTerrestrial Physics, vol. 3, pp. 72- 73, Nauka, Moscow, 1976.

Polyakov, S. V. and Rapoport, V. O.: Ionospheric Alfvén resonator, Geomagnetism and Aeronomy, $21(5), 816-822,1981$.

Robinson, T. R., Strangeway, R. J., Wright, D. M., Davies, J. A., Horne, R. B., Yeoman, T. K., Stocker, A. J., Lester, M., Rietveld, M. T., Mann, I. R., Carlson, C. W., and McFadden, J. P, FAST observations of ULF waves injected into the magnetosphere by means of modulated RF heating of the auroral electrojet, Geophys. Res. Lett., 27, 3165-3168, 2000.

Robinson T. R., T. K. Yeoman, R. S. Dhillon, M. Lester, E. C. Thomas, J. D. Thornhill, D. M. Wright, A. P. van Eyken and I. W. McCrea, First observations of SPEAR-induced artificial backscatter from CUTLASS and EISCAT Svalbard radars, Ann. Geophys., 24, 291-309, 2006.

Scoffield, H. C., T. K. Yeoman, T. R. Robinson, L. J. Baddeley, R. S. Dhillon, D. M. Wright, T. Raita, and T. Turunen, First results of artificial stimulation of the ionospheric Alfvén resonator at 78 N, Geophys. Res. Lett., 33, L19103, doi:10.1029/2006GL027384, 2006.

Semenova, N. V., A. G. Yahnin, A. N. Vasiliev, S. P. Noskov, and A. I. Voronin, First observations of the electromagnetic noise spectral resonance structures in the range of $0.1-4 \mathrm{~Hz}$ in the polar cap region (Barentsburg, Spitsbergen), in Complex Investigations of Spitsbergen Nature, Kol'skiy Sci. Cent., Russ. Acad. of Sci., Apatity, Russia., vol. 5, pp. 120 - 131, 2005 (in Russian).

Trakhtengerts, V. Y., P. P. Belyaev, S. V. Polyakov, A. G. Demekhov, and T. Bosinger, Excitation of Alfvén waves and vortices in the ionospheric Alfvén resonator by modulated powerful radio waves, J. Atmos. Sol. Terr. Phys., 62, 267-276, 2000.

\section{Figure captions}

Figure 1. Power spectral density for 18:00-18:40 UT from the pulsation magnetometer at Sodankylä, Finland on 4 October 1998 for window and slip size of 100 s. This is the average of 24 power spectra, smoothed with a boxcar of width 3 (Hebden et al., 2005).

Figure 2. Diagram showing an Alfvén wave's perpendicular electric field for the first 3 eigenmodes trapped in the IAR for the high conductivity ionosphere (panel (a)) and low conductivity (panel (b)) ionosphere. The lower red rectangles represent the lower IAR boundary and the blue rectangle represents the upper IAR boundary. The background magnetic field is directed downwards for the high latitude northern hemisphere regions. Panel a) for a high conductivity ionosphere shows a node 
at the lower IAR boundary. Panel b) for a low conductivity ionosphere displays an antinode at the lower IAR boundary (Hebden et al., 2005).

Figure 3. Upper panel: Naturally-occurring SRS signatures measured at Barentsburg. Averages of 20 spectra (each with $40 \mathrm{~Hz}$ sampling and a 1024 point FFT analysis giving a frequency resolution of $0.04 \mathrm{~Hz}$ ) results in a $8.5 \mathrm{~min}$ FFT window, which is slipped by $25.6 \mathrm{~s}$ in the dynamic spectrum, and normalised to $3 \mathrm{~Hz}$ peak power. The lower panel presents an autocorrelation analysis of the spectra, revealing the SRS harmonic separation as a function of time.

Figure 4. Average amplitude of the artificial magnetic signal as observed on the ground in the vicinity of the heater as a function of the modulation frequency of the HF power (Stubbe, 1996).

Figure 5. Dynamic spectrum (power spectral density in arbitrary units as a function of time and frequency) of the H-component magnetic disturbances artificially-stimulated by the EISCAT heater as observed at TRO during 16 November 1993 (Bösinger et al., 2000).

Figure 6. Peaks in the downward differential electron energy flux data from the FAST satellite (dashed least-squares regression lines) compare well to the theoretical energy shift $|\Delta E|$ that electrons would have after travelling through a region of localised parallel electric field (solid colour lines) for a power law energy distribution. Panel (a) shows data points for each peak in the signal, with the log of the phase space density plotted against the log of the electron energy, with the associated least-squares regression line (dashed lines). Uncertainty in the base flux data points (black triangles), due to the signal noise amplitude, are shown as error bars on panel (a). Panels (b)(d) show the phase space density expected by shifting the power law particle distribution from the base level (black solid line) by the $\Delta E$ displayed above each panel (solid coloured lines). Uncertainty in the peak phase space density data points (coloured diamonds), are shown on panels (b), (c), and (d) for peaks 1, 2 and 3, respectively (Cash et al., 2002).

Figure 7. A summary plot of BAR pulsation magnetometer during IAR artificial-stimulation experiments at SPEAR. ( $a$ and $b$ ) Fourier power of data at frequencies between 0.5 and $5.0 \mathrm{~Hz}$ as a fraction of the peak power at $3 \mathrm{~Hz}$ as it varies with time. Each pixel represents an average of 20 unique spectra, each calculated from a 1024 point (25.6 seconds) window with a 256 point (6.4 seconds) slip between pixels. This gives a time resolution of 6.4 seconds and a frequency resolution of $0.04 \mathrm{~Hz}$. Figure $6 \mathrm{a}$ shows $\mathrm{H}$ component data while Figure $6 \mathrm{~b}$ shows D component data. The D component peak was 1.6 times the magnitude of the $\mathrm{H}$ component peak. The bar at the top of the figure and the vertical dashed lines indicate when the heater was turned on (red) (Scoffield et al., 2006). 


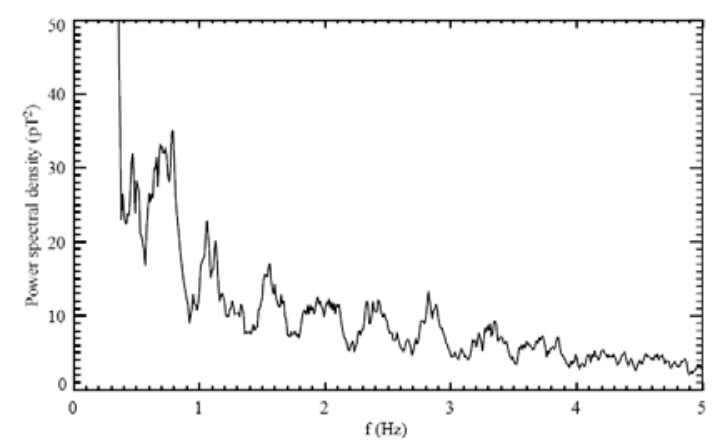

Figure 1 
a)

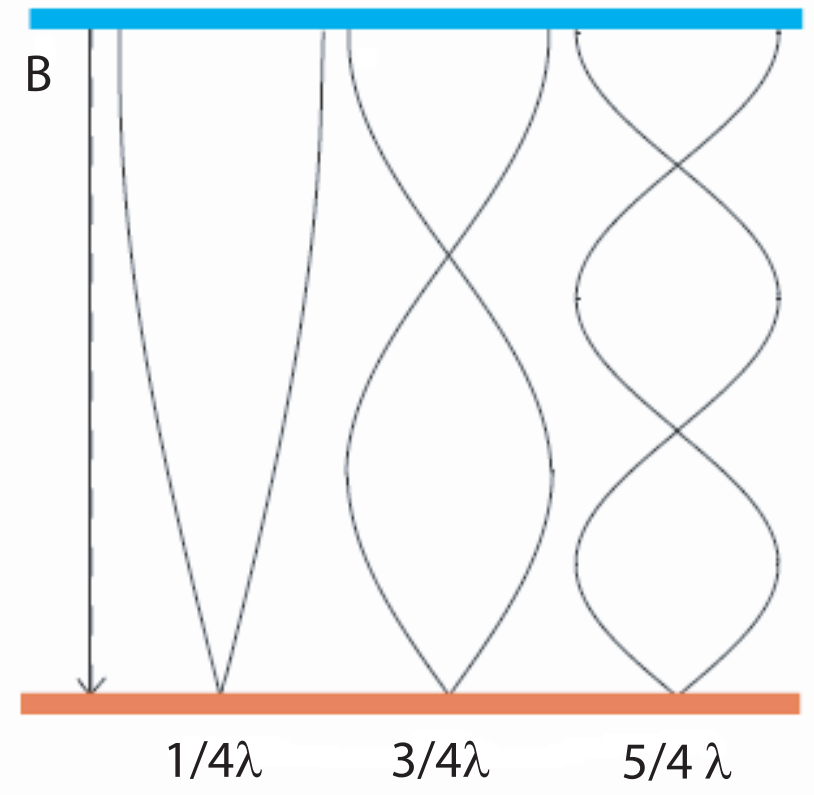

b)

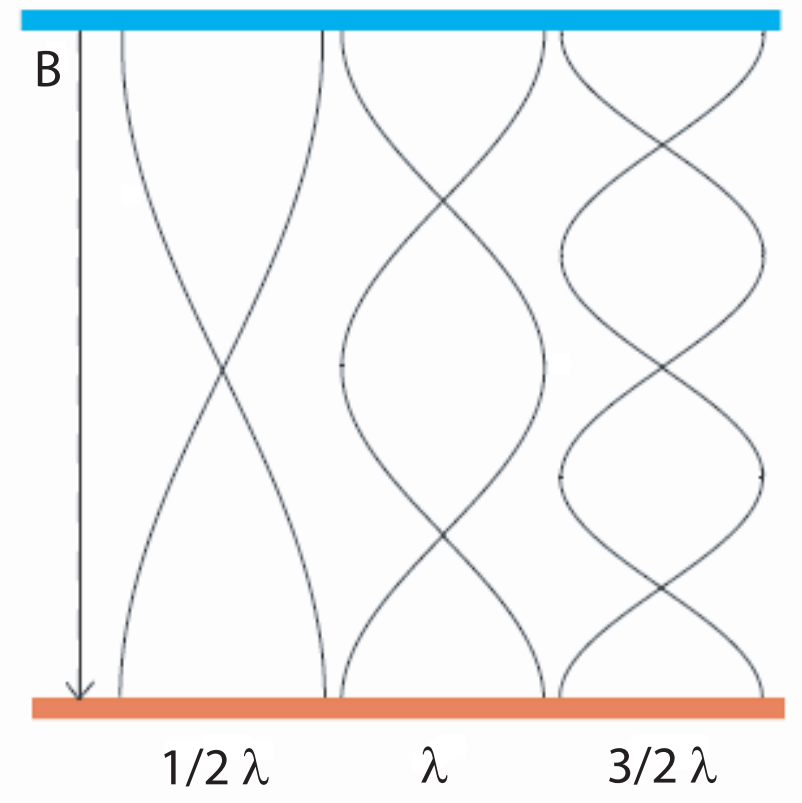



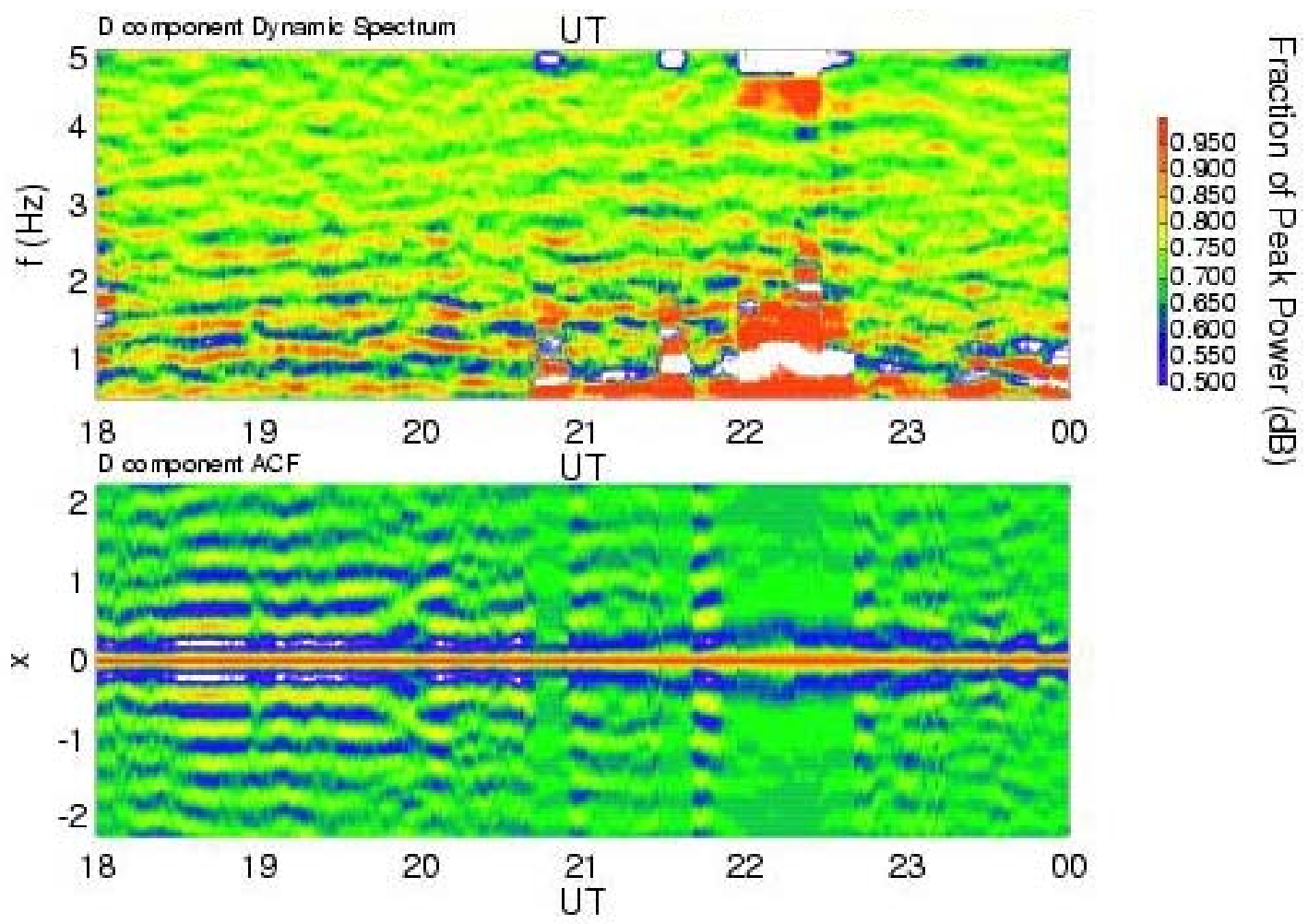

Figure 3 


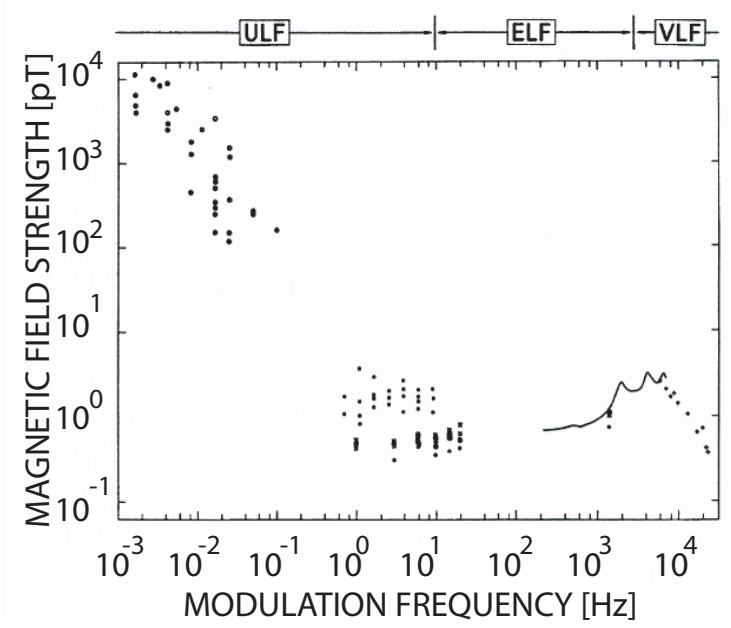

Figure 4 
TRO/H k1: 1993/11/16

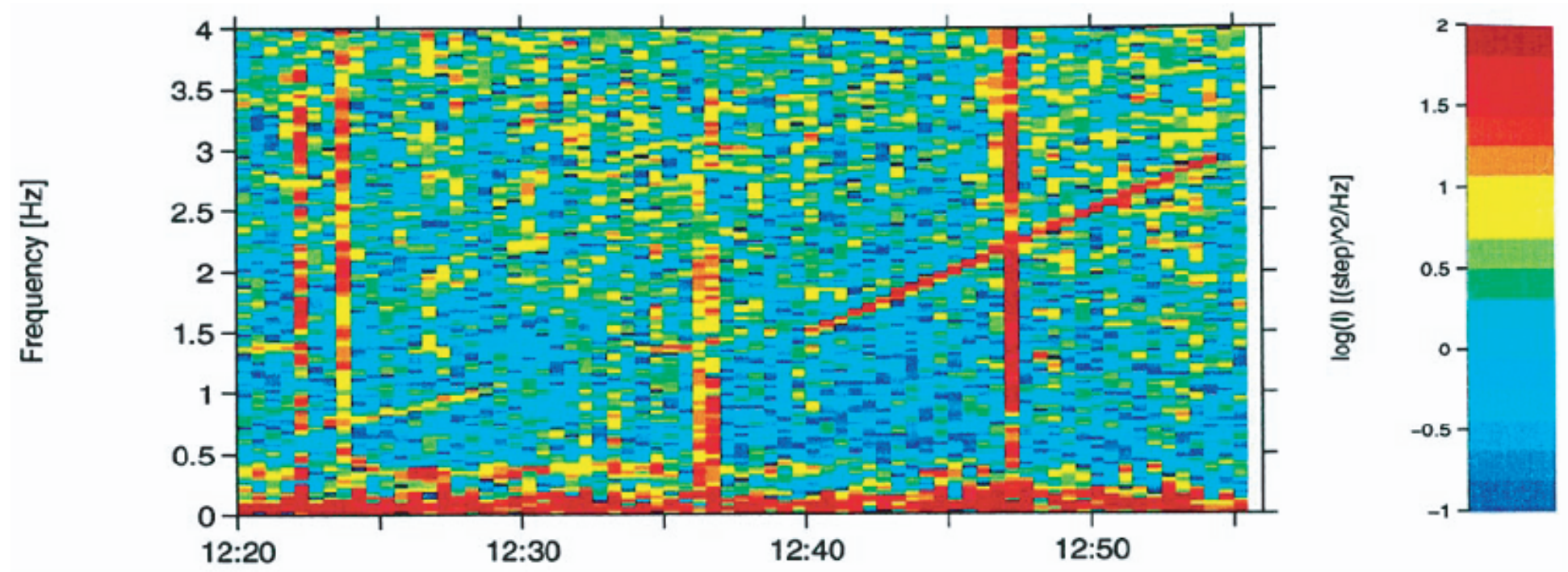


Data: peaks and base fluxes

a.

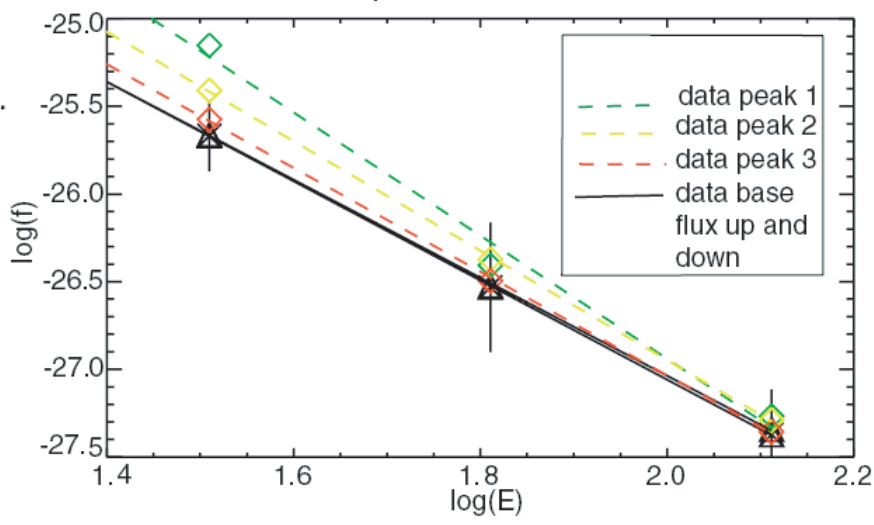

Peak 2: $\Delta \mathrm{E}=7.5 \mathrm{eV}$

c.

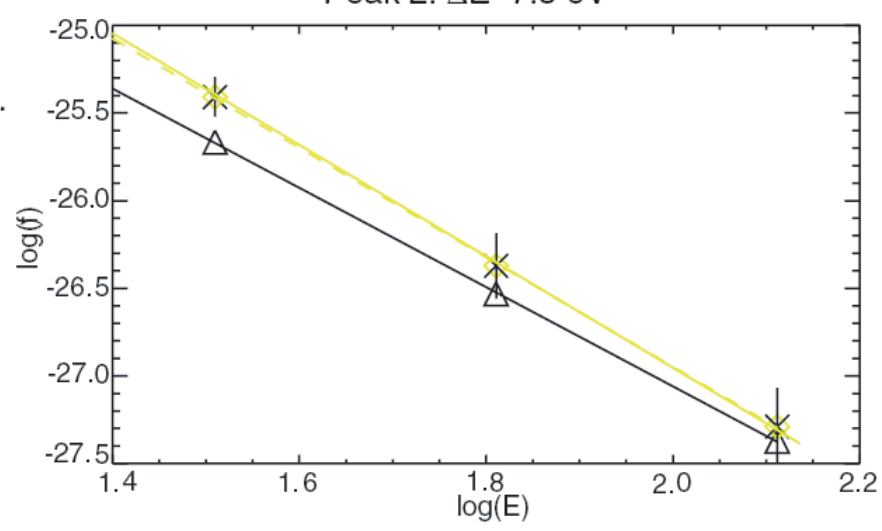

Peak 1: $\Delta \mathrm{E}=11 \mathrm{eV}$

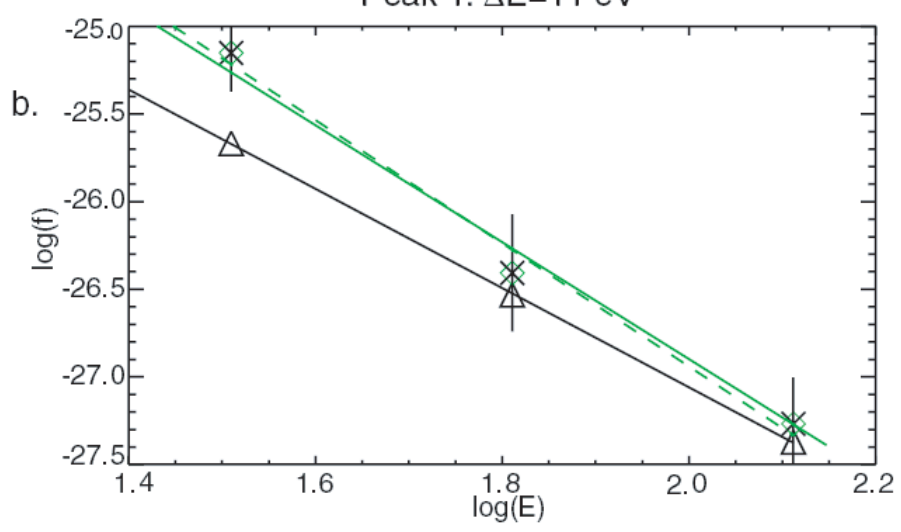

Peak 3: $\Delta \mathrm{E}=2.2 \mathrm{eV}$

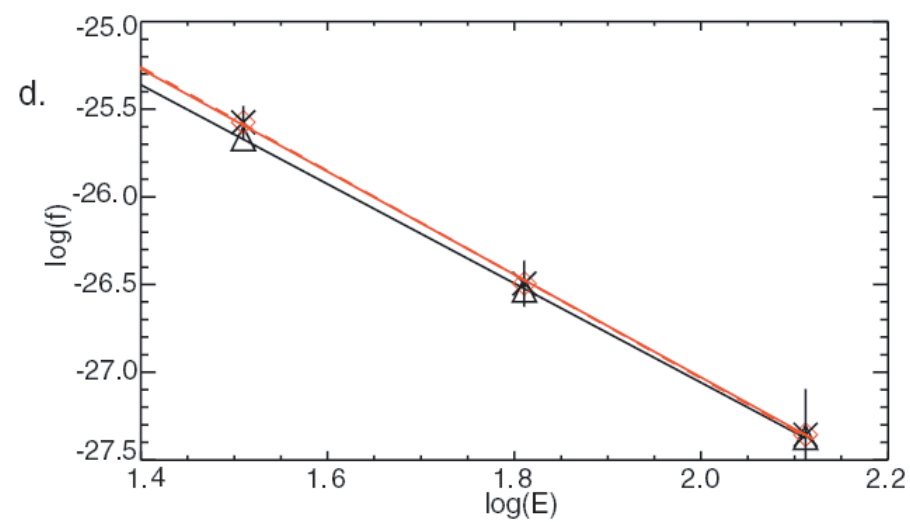

Figure 6 


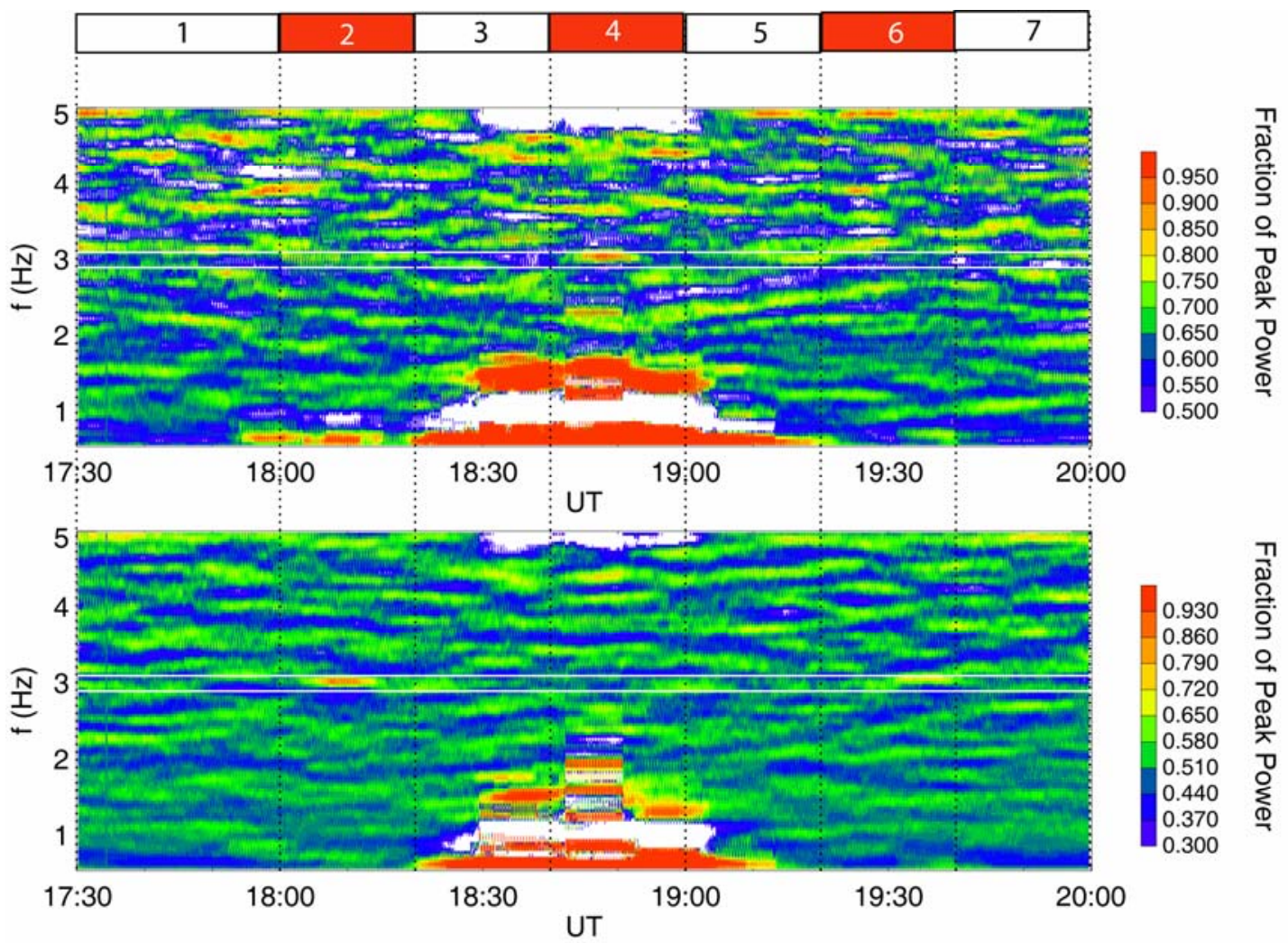

Figure 7 